\title{
MAGNETISM AND FERROELECTRICITY
}

\section{Digital Magnetic Heterostructures Based on Si and Fe}

\author{
M. M. Otrokov ${ }^{a}$ *, S. A. Ostanin ${ }^{b}$, A. Ernst ${ }^{b}$, V. M. Kuznetsov ${ }^{a}$, and E. V. Chulkov ${ }^{c}$, \\ ${ }^{a}$ Tomsk State University, pr. Lenina 36, Tomsk, 634050 Russia \\ *e-mail:otrokov@phys.tsu.ru \\ ${ }^{b}$ Max-Planck-Institut für Mikrostrukturphysik, Weinberg 2, Halle, D-06120 Germany \\ ${ }^{c}$ Donostia International Physics Center (DIPC), Paseo Manuel de Lardizabal 4, \\ Donostia-San Sebastián, Basque Country, 20018 Spain \\ ${ }^{d}$ Departamento de Física de Materiales and Centro de Física de Materiales (CFM) \\ CSIC-Universidad del País Vasco/Euskal Herriko Unibertsitatea (University of the Basque Country), \\ Facultad de Ciencias Quimicas, Universidad del País Vasco/Euskal Herriko Unibertsitatea \\ (University of the Basque Country) Barrio Sarriena s/n, Donostia-San Sebastián, Basque Country, 48940 Spain
}

Received November 18, 2009

\begin{abstract}
This paper reports on the results of ab initio calculations of the electronic and magnetic properties of Si digital heterostructures doped with a Fe monolayer of the substitutional or interstitial type. It has been revealed that, after the structural relaxation, heterostructures of both types exhibit a two-dimensional metallic behavior and a ferromagnetic ordering in the range of Si spacer thicknesses up to 19 atomic layers. The magnetization and spin polarization at the Fermi level for the heterostructures with an Fe monolayer of the substitutional type are two times higher than those for the system with an Fe monolayer of the interstitial type.
\end{abstract}

DOI: $10.1134 / \mathrm{S} 1063783410080184$

\section{INTRODUCTION}

Experimental and theoretical investigations of magnetic semiconductors are one of the most important fields of condensed matter physics from both technological and fundamental points of view. Magnetic semiconductors make it possible to control not only the charge of carriers, which underlies electronics, but also the spin of charge carriers. Therefore, they can have various applications. The majority of modern investigations in this field have been devoted to the study of GaAs-based systems. However, magnetic materials based on Si can appear to be more useful in practice, because it is Si that is the main material of electronics. The study of magnetic materials that can be easily integrated with Si electronic materials seems to be a very important problem. In this respect, $a b$ initio investigations are growing in significance, which is especially evident for the so-called digital magnetic heterostructures $(\mathrm{DMH})$ that consist of monolayers of magnetic transition metals in semiconductor films [1]. The DMH based on delta doping allow one to achieve magnetic impurity concentrations in semiconductors that considerably exceed the equilibrium solubility limit. The high degree of ordering of $\mathrm{DMH}$ and the perfectness of interfaces favor magnetic ordering. In the ferromagnetic (FM) case, the high concentration of magnetic atoms leads to a considerable increase in the Curie temperature [2]. Moreover, as follows from ab initio calculations, the DMH can have a higher spin polarization as compared to conventional ferromag- nets [3-5], thus representing ideal materials for spin injection. The corresponding heterostructures have already been prepared experimentally in GaAs/Mn [2, 6] and $\mathrm{GaSb} / \mathrm{Mn}[7,8]$ materials.

At present, investigations of Si DMH have been predominantly associated with the doping by manganese because of its considerable magnetic moment, i.e., approximately $2.3 \mu_{\mathrm{B}}$ in the $\alpha$ structure (see theoretical studies in $[4,5]$ and experimental investigations in [9]). Since Fe atoms also have a substantial magnetic moment $\left(2.2 \mu_{\mathrm{B}}\right.$ in the $\alpha$ (body-centered cubic) structure), it is also expedient to investigate $\mathrm{DMH}$ based on Si and Fe. Recently, Uspenskii and Kulatov [10] investigated the discrete magnetic heterostructure consisting of the $\mathrm{Fe}$ monolayer in Si by using the augmented plane wave method in the generalized gradient approximation to the exchange-correlation functional. The Fe monolayer was formed by means of the substitution of $\mathrm{Fe}$ atoms for $\mathrm{Si}$ atoms. In [10], it was demonstrated that, per two Fe atoms, the antiferromagnetic (AFM) configuration is more favorable than the FM configuration by $30 \mathrm{meV}$. It should be noted that no structural relaxations were performed in [10], even though their inclusion can result in a change in the "magnetic behavior" of the discrete magnetic heterostructure.

$\mathrm{Up}$ to now, $\mathrm{Si} / \mathrm{Fe}$ heterostructures with an interstitial monolayer (Fe atoms occupy tetrahedral interstices in the Si lattice) have never been investigated. Therefore, in this work, we present the ab initio inves- 


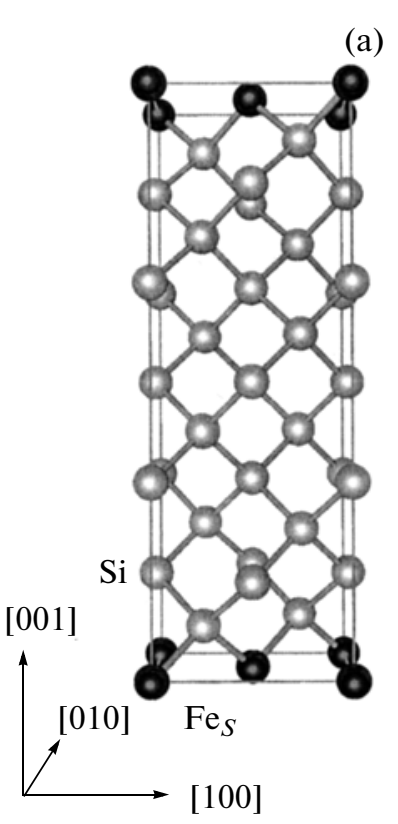

Fig. 1. Unit cells of Si heterostructures with an Fe monolayer of (a) the substitutional type $\mathrm{Si}_{N} / \mathrm{Fe}_{S}$ and (b) the interstitial type $\mathrm{Si}_{N} / \mathrm{Fe}_{I}$ for $N=11$. The figure was prepared with the Balls and Sticks program [11].

tigation of the electronic structure and magnetic properties of Si DMH doped with an Fe monolayer of the substitutional $\left(\mathrm{Fe}_{S}\right)$ or interstitial $\left(\mathrm{Fe}_{I}\right)$ type with taking into account the structural relaxations.

\section{DESCRIPTION OF THE MODEL AND COMPUTATIONAL METHOD}

The DMH were simulated using $\mathrm{Si}_{N} / \mathrm{Fe}_{S}$ and $\mathrm{Si}_{N} / \mathrm{Fe}_{I}$ supercells, which consisted of an Fe monolayer and a Si spacer with the thickness varying from $N$ $=7$ to 19 atomic layers. The basis vectors of the unit cell $\mathbf{a}, \mathbf{b}$, and $\mathbf{c}$ were directed along the [100], [010], and [001] axes, respectively, and their magnitudes were $a=b=a_{0}$ and $c=(N+1) a_{0} / 4$, where $a_{0}=5.46 \AA$ is the optimized lattice constant of bulk $\mathrm{Si}$ with a diamond structure. Figure 1 shows the $\mathrm{Si}_{11} / \mathrm{Fe}_{S}$ and $\mathrm{Si}_{11} / \mathrm{Fe}_{I}$ heterostructures. The calculations were performed in the framework of the density functional theory in the generalized gradient approximation to the exchange-correlation energy [12] with the use of the plane-wave basis set and the projector-augmented wave (PAW) pseudopotentials [13], as implemented in the VASP code [14-17]. The relaxation was carried out using the basis set of plane waves with energies $<500 \mathrm{eV}$ and the $6 \times 6 \times N_{z} \Gamma$-centered k-point mesh [18], where $N_{z}=3,2,2$, and 1 for the DMH consisting of $8,12,16$, and 20 layers, respectively. The use of the denser mesh $8 \times 8 \times N_{z}$ in the Brillouin zone leads to a

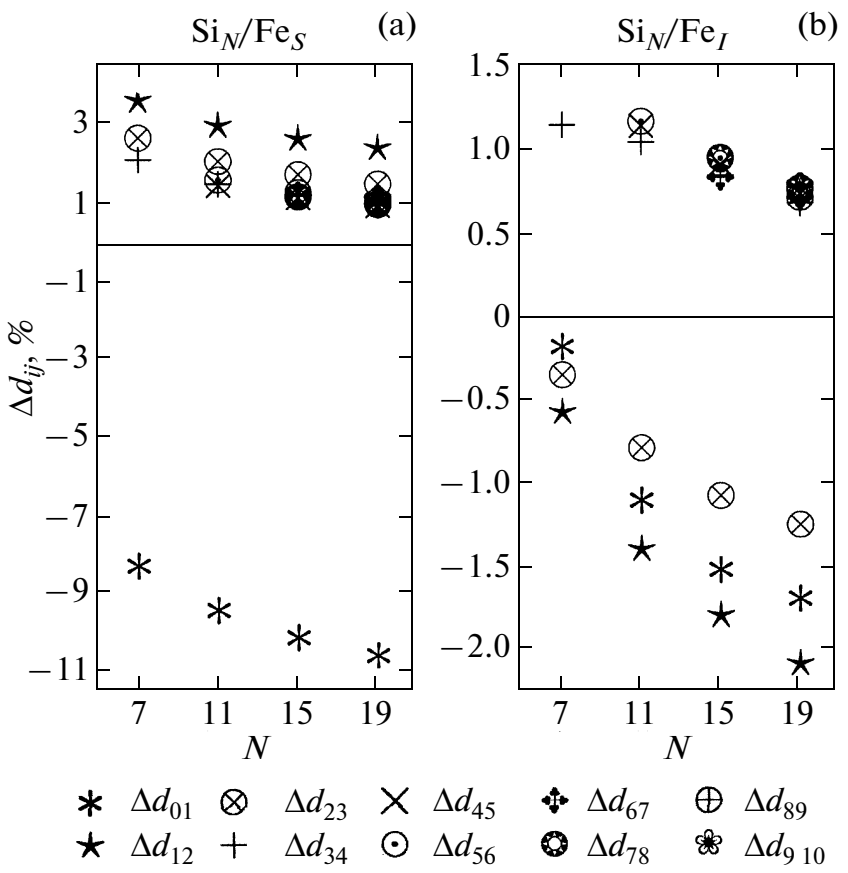

Fig. 2. Relative changes in the interplanar distances $\Delta d_{i j}$ after the performance of the structural relaxation in the (a) $\mathrm{Si}_{N} / \mathrm{Fe}_{S}$ and (b) $\mathrm{Si}_{N} / \mathrm{Fe}_{I}$ systems. The Fe monolayer has number 0 .

change in the total energy by no more than $1 \mathrm{meV}$ per atom. The structural relaxation was performed until the forces at each atom in the superlattice became $<0.01 \mathrm{eV} / \AA$. The densities of states were calculated using the $12 \times 12 \times N_{z}\left(N_{z}=6,4,3,2\right)$ meshes.

\section{RESULTS AND DISCUSSION}

\subsection{Structural Relaxation}

The results of the calculations of the structural relaxation in the $\mathrm{Si}_{N} / \mathrm{Fe}_{S}$ and $\mathrm{Si}_{N} / \mathrm{Fe}_{I}$ systems along the [001] direction are presented in Fig. 2. In the course of relaxation, each plane of the (001) type moves as a whole (intraplanar atomic displacements along the [001] direction are negligible and are of the order of $10^{-2} \%$ ), whereas the plane containing the $\mathrm{Fe}$ monolayer and the $(N+1) / 2$ th $\mathrm{Si}$ atomic layer remain fixed due to the periodicity of the cell. It can be seen from Fig. 2a that, during the relaxation in all $\mathrm{Si}_{N} / \mathrm{Fe}_{S}$ systems, the distance $\Delta d_{01}$ between the $\mathrm{Fe}_{S}$ monolayer and the nearest Si layers decreases. With an increase in $N$ from 7 to 19 , the quantity $\Delta d_{01}$ decreases from -8.3 to $-10.5 \%$. In this case, the $\mathrm{Fe}-\mathrm{Si}$ interatomic distance is approximately equal to $2.28 \AA$, which is very close to the experimentally observed distance $\mathrm{Fe}-\mathrm{Si}$ equal to $2.29 \AA$ in the $B 20$ structure [19]. The interatomic distance specified for the $\mathrm{Fe}-\mathrm{Si}$ pair before the relaxation (it corresponds to the distance between the nearest neighbors in perfect $\mathrm{Si}$ ) in our calculations 

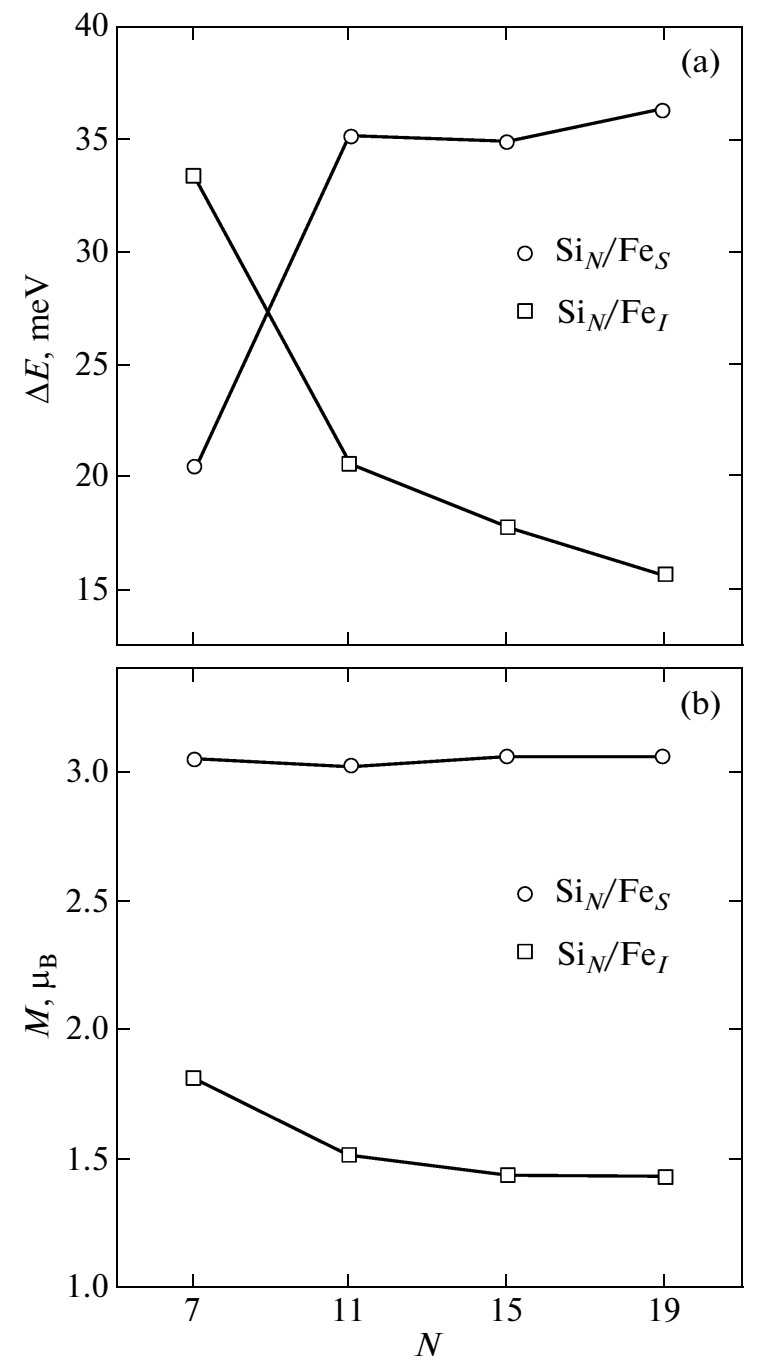

Fig. 3. (a) Difference between the energies of the AFM and FM configurations $\Delta E=E_{\mathrm{AFM}}-E_{\mathrm{FM}}$ per two Fe atoms as a function of the spacer thickness $N$. (b) Dependences of the net magnetic moment for the $\mathrm{Si}_{N} / \mathrm{Fe}_{S}$ and $\mathrm{Si}_{N} / \mathrm{Fe}_{I} \mathrm{FM}$ configurations.

amounts to $2.36 \AA$. Therefore, the decrease in the distance between the $\mathrm{Fe}_{S}$ monolayer and the nearest $\mathrm{Si}$ atomic planes in the $\mathrm{Si}_{N} / \mathrm{Fe}_{S}$ system is associated with the formation of the chemical bond length between the $\mathrm{Fe}$ and $\mathrm{Si}$ atoms. These atoms tend to form the bond with the length close to the length of the bond length between the same atoms in the conventional FeSi compound. Figure $2 b$ illustrates the relaxation in the system with the $\mathrm{Fe}_{I}$ monolayer. It can be seen from this figure that the changes in the interplanar distances during the relaxation are considerably smaller than those in the system with the substitutional monolayer: $\Delta d_{01}=-1.7 \%$ for the spacer thickness $N=19$. Most likely, these insignificant relaxations are a simple consequence of the fact that the addition of Fe atoms to tetrahedral interstitials (actually, the addition of the material to a fixed volume) inevitably leads to the repulsion of the neighboring layers. This effect competes with the mechanism of formation of a shortened bond length between the Fe and Si atoms. Nonetheless, an increase in the spacer thickness results in an increase in the interplanar displacements. It is evident that the stresses caused by the incorporation of the $\mathrm{Fe}$ monolayer are redistributed between a larger number of layers in the heterostructure and the tendency of the $\mathrm{Fe}$ and Si atoms toward the formation of the bond with the length close to the bond length in the bulk FeSi compound is enhanced.

\subsection{Magnetic Ordering}

It can be seen from Fig. 3a that, for Si spacer thicknesses $7 \leq N \leq 19$, both systems $\mathrm{Si}_{N} / \mathrm{Fe}$ are ordered ferromagnetically. It is interesting to note that the quantity $\Delta E$ changes substantially in the range from $N=7$ to 11 layers and then varies rather weakly with a further increase in the spacer thickness. This indicates that the observed ferromagnetism has a two-dimensional character, because the contribution of spacer exchange interaction to the total energy becomes negligible for $N \geq 11$. Consequently, the formation of the FM order is determined by the direct exchange interaction between electrons of Fe atoms of the same monolayer, which are located at a distance of $3.86 \AA$ from each other. Since the magnetic interactions along the [001] direction are weak, no change in the magnetic order in the ranges of thicknesses $N(7,11),(11,15)$, and $(15$, $19)$ is expected in the $\mathrm{Si}_{N} / \mathrm{Fe}$ systems. Previously, Uspenskii and Kulatov [10] investigated the DMH based on different semiconductors ( $\mathrm{Si}, \mathrm{Ge}, \mathrm{GaAs}$, $\mathrm{GaSb}, \mathrm{GaN}$ ) and transition metals ( $\mathrm{Cr}, \mathrm{Mn}, \mathrm{Fe}, \mathrm{Co}$ ) and revealed that the $\mathrm{Si} / \mathrm{Fe}_{S}$ system is antiferromagnetically ordered and that $\Delta E=-30 \mathrm{meV}$ per two $\mathrm{Fe}$ atoms. All DMH were studied without performing structural relaxations and only for one spacer thickness equal to 23 layers for the DMH with a diamond structure. Actually, in some cases, the inclusion of the structural relaxation does not lead to a qualitative change in the behavior of the system and, hence, can be omitted, for example, in $\mathrm{Si} / \mathrm{Mn}_{S}$ [4], where the distance $d_{01}$ changes insignificantly. However, the delta doping of $\mathrm{Si}$ with $\mathrm{Fe}$ with the substitutional type results in a decrease in the distance $d_{01}$ by $\sim 10 \%$, which substantially affects the type of magnetic ordering.

\subsection{Electronic Structure}

In crystalline $\mathrm{Si}$, with a diamond structure, the substitutional defects $\mathrm{Fe}_{S}$ and the interstitial defects $\mathrm{Fe}_{I}$ are located in the tetrahedral environment of four $\mathrm{Si}$ nearest neighbors. In this case, the fivefold degenerate $d$ levels of Fe atoms are split by the crystal field into the doubly degenerate level $e$ and the triply degenerate level $t_{2}$ [20]. The electronic orbitals of the Fe atoms 
and their Si nearest neighbors are hybridized. According to the molecular orbital theory, the hybridization can occur only for states with identical symmetry [21]; in our case, these are the $\mathrm{Si} t_{2}(p)$ and $\mathrm{Fe} t_{2}(d)$ states.

The total and partial densities of states (DOS) calculated for the $\mathrm{Si}_{19} / \mathrm{Fe}_{S}$ and $\mathrm{Si}_{19} / \mathrm{Fe}_{I}$ systems are shown in Fig. 4. The DOS for the other $\mathrm{Si}_{N} / \mathrm{Fe}_{S}$ and $\mathrm{Si}_{N} / \mathrm{Fe}_{I}$ systems, except for specially noted differences, are very similar to those shown in Fig. 4 and, therefore, are not presented in our work. As can be seen from Fig. 4a (see the upper panel), the $\mathrm{Si}_{19} / \mathrm{Fe}_{S}$ system exhibits a metallic DOS with the spin polarization at the Fermi level $P=|n \uparrow-n \downarrow| /(n \uparrow+n \downarrow)=0.6$ (or 60\%), where $n \uparrow=n \uparrow\left(E_{\mathrm{F}}\right)$ and $n \downarrow=n \downarrow\left(E_{\mathrm{F}}\right)$ are the differential DOS for spins up and spins down, respectively. In the majority-spin channel (solid lines), the states in the valence band between -2.5 and $1.0 \mathrm{eV}$ are characterized by a strong $\mathrm{Fe} d-\mathrm{Si}(1) p$ hybridization, which leads to "metallization" of local DOS of the Si atoms of the nearest layers (see panels $\mathrm{Fe} t_{2}(d)$ and $\mathrm{Si}(1), \mathrm{Si}(2)$, etc.). A considerable contribution to the valence band is also made by the $e(d)$ states located $1.5 \mathrm{eV}$ below the Fermi level. In the vicinity of the upper edge of the valence band, the contributions of the $\mathrm{Fe} d$ states and Si $p$ states are insignificant, which results in a low total DOS at the Fermi level in the majority-spin channel. In the conduction band, the fraction of the $\mathrm{Fe} d$ states with spin up is negligible and the contribution of the $\mathrm{Si}$ $s$ and $\mathrm{Si} p$ states increases away from the Fermi level $\left(E>E_{\mathrm{F}}\right)$ (compare panels Total and $\mathrm{Si}($ bulk) in Fig. 4a). In the minority-spin channel (dotted lines), the largest contribution to the conduction band in the range $0.5 \mathrm{eV}$ above the Fermi level is made by the Fe $e(d)$ orbitals. It is these $\mathrm{Fe} e(d)$ orbitals that provide a high spin polarization of the $\mathrm{Si}_{19} / \mathrm{Fe}_{S}$ discrete magnetic heterostructure at the Fermi level, because the total DOS in the vicinity of the upper edge of the valence band of the minority-spin channel is rather low. The $t_{2}(d)$ orbitals make a smaller contribution to the DOS at the Fermi level, because they are predominantly located in the range $2 \mathrm{eV}$ below the Fermi level $E_{\mathrm{F}}$, where the $\mathrm{Fe} d-\mathrm{Si}(1) p$ hybridization is also strong. Each $\mathrm{Fe}_{S}$ atom has a magnetic moment equal to $1.53 \mu_{\mathrm{B}}$. The magnetic moments induced at the four nearest atoms $\mathrm{Si}(1)$ are negative and amount to $0.06 \mu_{\mathrm{B}}$, the magnetic moments of the $\mathrm{Si}(2)$ atoms of the second coordination sphere are equal to $0.02 \mu_{\mathrm{B}}$, and the magnetic moments of the father atoms located in the subsequent layers are smaller than $0.01 \mu_{\mathrm{B}}$. With allowance made for a magnetic moment of $0.13 \mu_{\mathrm{B}}$ outside the Wigner-Seitz spheres, the net magnetic moment per unit cell is equal to $3.06 \mu_{\mathrm{B}}$ (Fig. 3b).

The DOS for the $\mathrm{Si}_{19} / \mathrm{Fe}_{I}$ system are shown in Fig. $4 \mathrm{~b}$. The partial DOS in the range from -3 to $1 \mathrm{eV}$ for both spin channels are characterized by a strong hybridization of the Fe $d$ states with the $p$ states of the $\mathrm{Si}(1), \mathrm{Si}(2)$, and $\mathrm{Si}(0)$ atoms, to which the distances are equal to $2.35,2.65$, and $2.73 \AA$, respectively. In this case, the partial densities of $t_{2}(d)$ and $e(d)$ states acquire a more "volume-like" form as compared to those for the $\mathrm{Si}_{19} / \mathrm{Fe}_{S}$ system (compare, for example, the pronounced peak of the $e(d)$ states for the $\mathrm{Si}_{19} / \mathrm{Fe}_{S}$ system and wide bands of the $e(d)$ states for the $\mathrm{Si}_{19} / \mathrm{Fe}_{I}$ system in the minority-spin channels). In combination with an insignificant exchange splitting of the Fe $d$ states, this leads to the following results: (1) a relatively low (of the order of $20 \%$ ) spin polarization at the Fermi level and (2) a decrease in the magnetic moment at the $\mathrm{Fe}_{I}$ atoms (as compared to the $\mathrm{Si}_{19} / \mathrm{Fe}_{S}$ system) to $0.71 \mu_{\mathrm{B}}$. As a consequence, the magnetic moments induced at the nearest $\mathrm{Si}$ atoms are small (less than $\left.0.01 \mu_{\mathrm{B}}\right)$. With due regard for a magnetic moment of $0.01 \mu_{\mathrm{B}}$ outside the Wigner-Seitz spheres, the net magnetic moment per unit cell is equal to $1.43 \mu_{\mathrm{B}}$ (Fig. 3b). The factor responsible for the broadening of the Fe $d$ bands in the interstitial monolayer lies in a closer location of atoms and, hence, in a stronger interatomic interaction as compared to that in the plane of the $\mathrm{Fe}_{S}$ monolayer. Actually, in the case of the substitutional monolayer, the first neighbor of the $\mathrm{Fe}$ atom in the monolayer plane is also the $\mathrm{Fe}$ atom located at a distance of $3.86 \AA$. In the interstitial monolayer, the distance between the $\mathrm{Fe}$ atoms is also equal to $3.86 \AA$, but the $\mathrm{Si}(0)$ atom is located at a distance of $2.73 \AA$ (Fig. 1). Therefore, the atomic density in the plane containing the interstitial monolayer is higher than that in the substitutional layer plane. As a result, the arising higher electron concentration leads to a stronger interatomic interaction and hybridization of bands. The decrease in the exchange splitting of the $\mathrm{Fe}_{I} d$ subband as compared to the exchange splitting of the $\mathrm{Fe}_{S} d$ subband can be understood using the following simple explanation. The exchange splitting of the Fe $d$ levels in the $\mathrm{Si}_{19} / \mathrm{Fe}_{S}$ system is smaller than that in body-centered cubic $\mathrm{Fe}$, because the nearest neighbors of Fe atoms in the discrete magnetic heterostructure are nonmagnetic $\mathrm{Si}$ atoms. Since four additional nonmagnetic neighbors, i.e., $\mathrm{Si}(0)$ atoms, appear for the $\mathrm{Fe}$ atom in the $\mathrm{Si}_{19} / \mathrm{Fe}_{I}$ system, the exchange splitting of its $d$ levels becomes even smaller.

It should be noted that, in both the $\mathrm{Si}_{19} / \mathrm{Fe}_{S}$ system and the $\mathrm{Si}_{19} / \mathrm{Fe}_{I}$ system, all $\mathrm{Si}$ atoms of the first seven layers (the reference point corresponds to the $\mathrm{Fe}$ monolayer) make a contribution to the DOS in the region of the fundamental band gap. This contribution decreases with an increase in the distance from the $\mathrm{Si}$ atoms to the Fe monolayer. Beginning with the eighth layer, the local DOS for the Si atoms exhibit a band gap of approximately $0.6 \mathrm{eV}$ and the form corresponding to that of the local DOS of intrinsic bulk Si (see panel $\mathrm{Si}$ (bulk)). Consequently, both types of the DMH under consideration represent two-dimensional metals. It is clear that their conductivity along the [001] 


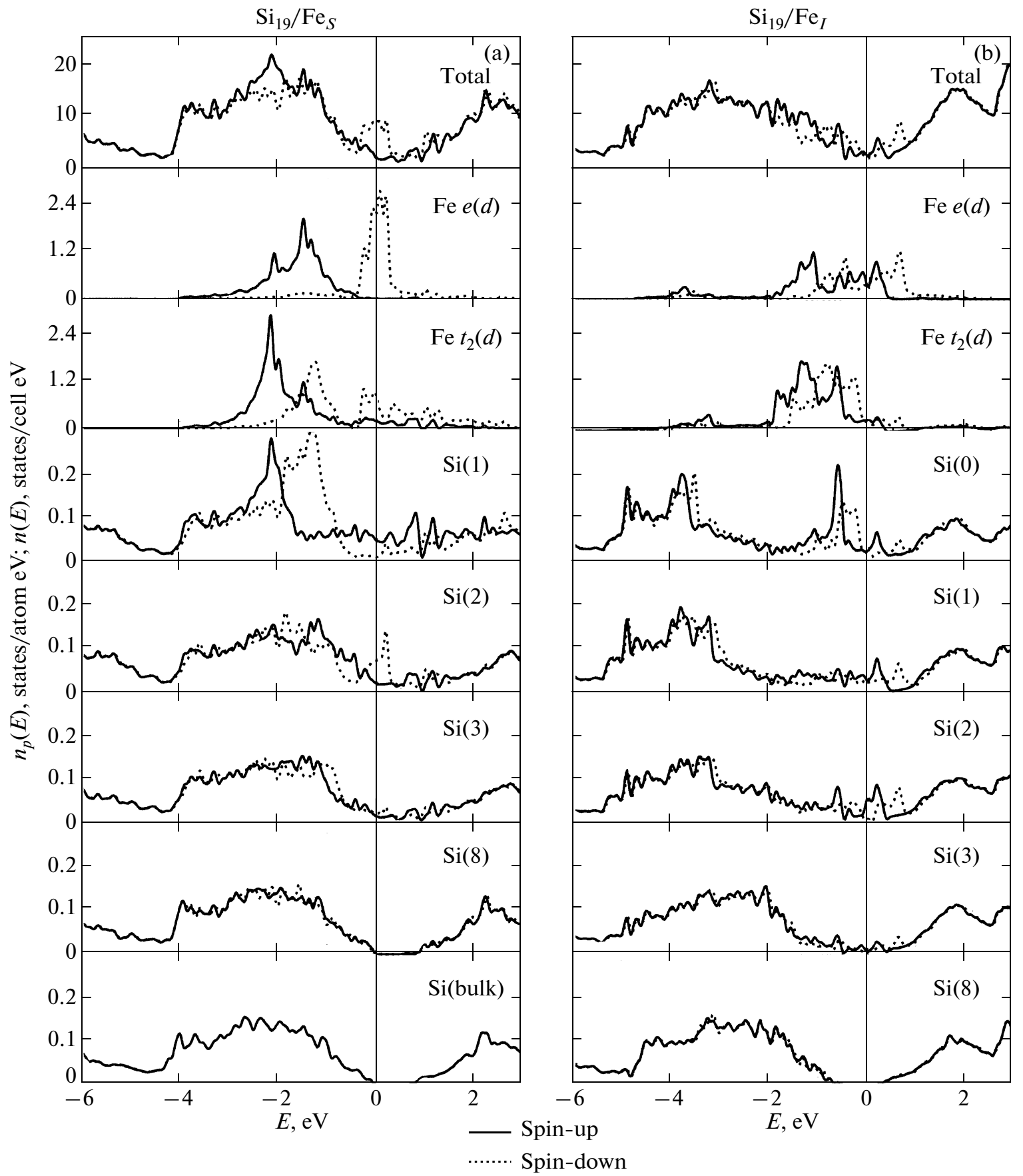

Fig. 4. Calculated total $n(E)$ and partial $n_{p}(E)$ DOS for the (a) $\mathrm{Si}_{19} / \mathrm{Fe}_{S}$ and (b) $\mathrm{Si}_{19} / \mathrm{Fe}_{I}$ systems. The Fermi level corresponds to zero energy. The $\mathrm{Si}(1), \operatorname{Si}(2), \operatorname{Si}(3)$, and $\mathrm{Si}(8)$ atoms are the $\mathrm{Si}$ atoms in the layers with numbers $1,2,3$, and 8 , respectively (the reference point corresponds to the Fe monolayer). For the $\mathrm{Si}_{19} / \mathrm{Fe}_{I}$ system, $\mathrm{Si}(0)$ indicates the atom located in the plane of the $\mathrm{Fe}_{I}$ monolayer. The $\mathrm{Si}$ (bulk) atom corresponds to the atom in intrinsic bulk Si with a diamond structure (in this case, the shown partial DOS is not spin-polarized). Partial DOS for all Si atoms represent the summarized contribution of the $s$ and $p$ states in the corresponding spin channels inside the Wigner-Seitz spheres.

direction is considerably lower than the conductivity in the plane doped with Fe. This property is retained for the DMH with thin Si spacers. Although, at $N=7$, none of the Si atoms have a DOS with a pronounced band gap, the conductivity of these DMH in the direc- tion perpendicular to the monolayer plane, as before, is substantially lower than that along this plane.

Figure 5 shows the band structures for the $\mathrm{Si}_{19} / \mathrm{Fe}_{S}$ and $\mathrm{Si}_{19} / \mathrm{Fe}_{I}$ systems along the $\Gamma-X-M-\Gamma-Z$ symme- 

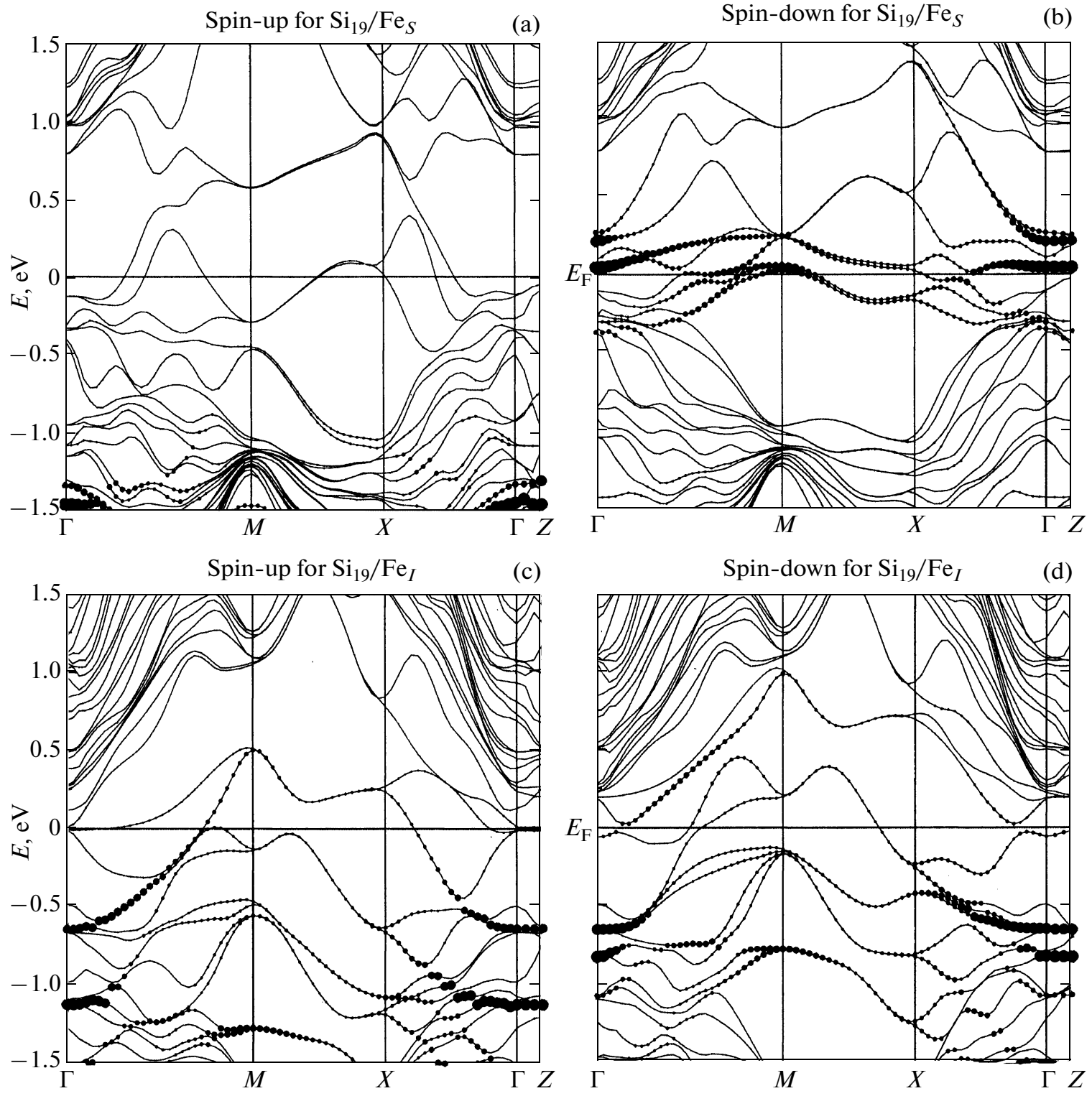

Fig. 5. Spin-polarized band structures of the (a, b) $\mathrm{Si}_{19} / \mathrm{Fe}_{S}$ and (c, d) $\mathrm{Si}_{19} / \mathrm{Fe}_{I}$ systems along the $\Gamma-M-X-\Gamma-Z$ direction of the Brillouin zone. The coordinates of the symmetry points of the Brillouin zone are as follows: $\Gamma=(0,0,0), M=(0.5,0.5,0), X=$ $(0.5,0,0)$, and $Z=(0,0,0.5)$. The size of symbols reflects the relative fraction of the $d$ states at the given $\mathbf{k}$ point.

try directions in the Brillouin zone. The $\Gamma-X-M-\Gamma$ and $\Gamma-Z$ directions, which lie in the $\mathbf{k}_{x}-\mathbf{k}_{y}$ and $\mathbf{k}_{z}$ planes of the Brillouin zone, respectively, describe the states in the plane of the $\mathrm{Fe}$ monolayer (i.e., in the (001) plane) and along the perpendicular [001] direction of the direct lattice. The size of symbols in the figures reflects the relative fraction of the $d$ states. In the majority-spin channel for the $\mathrm{Si}_{19} / \mathrm{Fe}_{S}$ system (Fig. 5a), three partially occupied bands provide a metallic conductivity. As was noted above, the states in the conduction band of the majority-spin channel are predominantly Si $t_{2}(p)$ in character, because the $e(d)$ and $t_{2}(d)$ states are located below the Fermi level (see panel Fe $t_{2}(d)$ in Fig. 4a). In the minority-spin channel, the conductivity also exhibits a metallic behavior. At the Fermi level and its vicinity, there are narrow bands in which the states are predominantly $e(d)$ in character (see panels Fe $e(d)$ and Fe $t_{2}(d)$ in Fig. 4). These states ensure a high spin polarization at the Fermi level of the system. Along the $\Gamma-Z$ direction, the larger the fraction of the $d$ states in the $p-d$ hybridized bands, the higher their localization. This is a consequence of the specific structure of the discrete magnetic heterostructure: along the [001] direction, the profile of the dopant distribution is a delta function. Therefore, along the $\Gamma-Z$ direction, the narrow impu- 
rity bands are located in contrast to the broadened bands located along the $\Gamma-X-M-\Gamma$ direction, which describe the states of electrons in the monolayer plane, where a two-dimensional metallic bonding appears. Moreover, in both spin channels, states at the Fermi level along the $\Gamma-Z$ direction are absent; the charge carriers are predominantly located in the vicinity of the $\mathrm{Fe}_{S}$ monolayer, which also confirms the twodimensional metallic behavior of the system under consideration.

The metallic conductivity in the $\mathrm{Si}_{19} / \mathrm{Fe}_{I}$ system is provided by three and two partially occupied bands in the majority-spin and minority-spin channels, respectively (Figs. 5c, 5d); in this case, it can be seen from Fig. $4 \mathrm{~b}$ that the conduction band contains the states of both symmetries $e(d)$ and $t_{2}(d)$. In the $\mathbf{k}_{x}-\mathbf{k}_{y}$ plane (the $\Gamma-X-M-\Gamma$ directions), the extent of the $p-d$ hybridized bands is larger, on average, than that in the $\mathrm{Si}_{19} / \mathrm{Fe}_{S}$ system, which indicates a more considerable interatomic interaction in the $\mathrm{Fe}_{I}$ monolayer. As regards the $\Gamma-Z$ direction, the bands with the dominance of the $d$ character are also strongly localized, as is the case in the $\mathrm{Si}_{19} / \mathrm{Fe}_{S}$ system, due to the delta profile of the dopant distribution in the [001] direction of the direct lattice. The $\mathrm{Si}_{19} / \mathrm{Fe}_{I}$ system, like the $\mathrm{Si}_{19} / \mathrm{Fe}_{S}$ system, has no states at the Fermi level along the $\Gamma-Z$ direction: the majority-spin channel has a pseudogap equal to $29 \mathrm{meV}$. This circumstance suggests that charge carriers are predominantly located in the vicinity of the $\mathrm{Fe}_{I}$ monolayer.

\section{CONCLUSIONS}

Thus, the $N$-layer Si DMH with an Fe layer of the substitutional or interstitial type have been investigated using ab initio calculations. It has been demonstrated that, in the study of the $\mathrm{Si}_{N} / \mathrm{Fe}_{S}$ system, it is very important to account for the structural relaxation, because the difference between the energies of the FM and AFM configurations $\Delta E$ is sensitive to a change in the distance between the $\mathrm{Fe}_{S}$ monolayer and the nearest $\mathrm{Si}$ atomic planes. Both types of the doped systems are ferromagnetically ordered over the entire range of Si spacer thicknesses $N$ under investigation. For $N \geq$ 11, the DMH under consideration exhibit a twodimensional FM order, which arises as a result of the direct exchange between the $\mathrm{Fe}$ atoms inside the monolayer. For $N=7$, there can occur insignificant exchange interactions between the neighboring $\mathrm{Fe}$ monolayers, whereas, at $N \geq 11$, their contribution to $\Delta E$ is negligible. In view of the decrease in the exchange splitting, the $d$ subbands with different spin directions in the $\mathrm{Si}_{N} / \mathrm{Fe}_{I}$ systems more strongly overlap as compared to the $\mathrm{Si}_{N} / \mathrm{Fe}_{S}$ systems. As a result, the net magnetic moment and the spin polarization in the $\mathrm{Si}_{N} / \mathrm{Fe}_{S}$ systems exceed the corresponding quantities in the $\mathrm{Si}_{N} / \mathrm{Fe}_{I}$ systems by a factor of more than two: $1.53 \mu_{\mathrm{B}}$ and $60 \%$ as compared to $0.71 \mu_{\mathrm{B}}$ and $20 \%$, respectively. According to our calculations and the data available in the literature [3-5], the two-dimensional metallic behavior is a property in common for DMH irrespective of a specific semiconductor matrix and a delta doping transition metal. This means that, in these systems, the conductivity in the direction perpendicular to the monolayer plane is substantially lower than the conductivity along this plane, because charge carriers are predominantly located in the vicinity of the transition metal monolayer.

The calculations were performed on the SKIF Cyberia computational cluster at the Tomsk State University (Tomsk, Russia).

\section{ACKNOWLEDGMENTS}

We would like to thank S.V. Eremeev and I.A. Nechaev for helpful discussion.

This study was supported in part by the University of the Basque Country (project GV-UPV, grant no. IT-366-07) and the Spanish Ministerio de Ciencia y Tecnología (MCyT) (grant no. FIS2007-06671C02-01).

\section{REFERENCES}

1. S. A. Crooker, D. A. Tulchinsky, J. Levy, D. D. Awschalom, R. Garcia, and N. Samarth, Phys. Rev. Lett. 75, 505 (1995).

2. A. M. Nazmul, T. Amemiya, Y. Shuto, S. Sugahara, and M. Tanaka, Phys. Rev. Lett. 95, 017201 (2005).

3. S. Sanvito, Phys. Rev. B: Condens. Matter 68, 054425 (2003).

4. M. C. Qian, C. Y. Fong, K. Liu, W. E. Pickett, J. E. Pask, and L. H. Yang, Phys. Rev. Lett. 96, 027211 (2006).

5. H. Wu, P. Kratzer, and M. Scheffler, Phys. Rev. Lett. 98, 117202 (2007).

6. R. K. Kawakami, E. Johnston-Halperin, L. F. Chen, M. Hanson, N. Guébels, J. S. Speck, A. C. Gossard, and D. D. Awschalom, Appl. Phys. Lett. 77, 2379 (2000).

7. X. Chen, M. Na, M. Cheon, S. Wang, H. Luo, B. D. McCombe, X. Liu, Y. Sasaki, T. Wojtowicz, J. K. Furdyna, S. J. Potashnik, and P. Schiffer, Appl. Phys. Lett. 81, 511 (2002).

8. G. B. Kim, M. Cheon, S. Wang, H. Luo, B. D. McCombe, X. Liu, Y. Sasaki, T. Wojtowicz, and J. K. Furdyna, Physica E (Amsterdam) 20, 355 (2004).

9. S. H. Chiu, H. S. Hsu, and J. C. A. Huang, J. Appl. Phys. 103, 07D110 (2008).

10. Yu. A. Uspenskii and E. T. Kulatov, J. Magn. Magn. Mater. 321 (7), 931 (2009). 
11. Balls and Sticks: Program Designed to Create 3D-Pictures and Animations of Crystals http://www.softbug.com/toycrate/bs/index.html; T. C. Ozawa and S. J. Kang, J. Appl. Crystallogr. 37, 679 (2004).

12. J. P. Perdew and W. Wang, Phys. Rev. B: Condens. Matter 45, 13244 (1992).

13. P. E. Blöchl, Phys. Rev. B: Condens. Matter 50, 17953 (1994).

14. G. Kresse and J. Hafner, Phys. Rev. B: Condens. Matter 47, 558 (1993); Phys. Rev. B: Condens. Matter 49, 14251 (1994).

15. G. Kresse and J. Furthmüller, Comput. Mater. Sci. 6, 15 (1996).
16. G. Kresse and J. Furthmüller, Phys. Rev. B: Condens. Matter 54, 11169 (1996).

17. G. Kresse and J. Joubert, Phys. Rev. B: Condens. Matter 59, 1758 (1999).

18. H. J. Monkhorst and J. D. Pack, Phys. Rev. B: Solid State 13, 5188 (1976).

19. R. Wartchow, S. Gerighausen, and M. Binnewies, Z. Kristallogr. - New Cryst. Struct. 212, 320 (1997).

20. F. A. Cotton, Chemical Applications of Group Theory (Wiley, New York, 1990).

21. S. Fudzinaga, Method of Molecular Orbitals (Iwanami Shoten, Tokyo, 1980; Mir, Moscow, 1983).

Translated by O. Borovik-Romanova 